\title{
Schulabsentismus - ein neuer Blick auf ein altes Phänomen
}

\author{
Inga Mau, Sebastian Messer, Katja vom Schemm
}

\section{Problemaufriss}

S chulabsentismus - Willkommen in der Begriffsdiffusion! Obwohl seit Ende der 90-er Jahre das Phänomen des Schulabsentismus in aller Munde ist, seine Zusammenhänge zur Jugenddelinquenz (BMI/BMJ, 2006) und zum schlechten Abschneiden in den PISA-Studien sowohl in der Wissenschaft als auch in den Medien thematisiert werden, besteht derzeit noch keine Einigkeit über eine einheitliche Definition. Manche Länder zählen entschuldigtes Fehlen dazu, andere nicht. Es liegt auf der Hand, dass ein weites Verständnis Vorteile hat, da es eine größere Gruppe zu den unregelmäßig die Schule Besuchenden zählen kann. Da sich die Kultus- und Bildungsministerien der Länder wegen der PISA-Studie und der verstärkten öffentlichen Berichterstattung im Zugzwang sehen, müssen sie nach präsentablen Gründen für das international vergleichsweise schlechte Abschneiden der deutschen SchülerInnen suchen. Dass ein wichtiger Aspekt in einem unregelmäßigen Schulbesuchsverhalten liegen könnte, scheint evident, da dieses doch in einem engen Zusammenhang mit schulischen Misserfolgen, einem drohenden sozialen Ausschluss (siehe II.) und sogar Delinquenz (siehe IV.) steht. Doch wie kann man unregelmäßiges Schulbesuchsverhalten bzw. Schulabsentismus definieren, operationalisieren und damit dem bisherigen wissenschaftlichen Diskurs zugänglich machen? Geht man davon aus, dass ein Schulhalbjahr etwa 100 Schultage hat, stellt sich beispielsweise die Frage, ab welcher Häufigkeit und in welcher Form (entschuldigte und/ oder unentschuldigte?) Fehlzeiten als problematisch angesehen werden müssen. Der nachfolgende Artikel gibt, gerade auch hinsichtlich der gesellschaftlichen Folgen und dem evi- denten Zusammenhang zu delinquentem Verhalten, einen Überblick über wesentliche Aspekte in der wissenschaftlichen Diskussion und verdeutlicht die Notwendigkeit einer einheitlichen Herangehensweise, intensiverer Ursachenforschung und mögliche Ansätze zur Entwicklung präventiver Maßnahmen bei Schulabsentismus.

\section{Forschungsstand und defini- torische Schwierigkeiten}

Bereits in den 70-er Jahren fand die Problematik beispielsweise mit den Veröffentlichungen von Preu $\beta$ (1978) und Hildeschmid, Meister, Sander und Schorr (1979) eine weitergehende Beachtung, der wissenschaftliche Diskurs verebbte jedoch bald. Erst durch die Untersuchungen von Wetzels, Mecklenburg, Wilmers, Enzmann und Pfeiffer (2000a) sowie Wetzels, Wilmers, Mecklenburg, Enzmann und Pfeiffer (2000b) erlebte die Schulabsentismus-Debatte - dann auch im Zuge des „Pisa-Schocks" - eine Renaissance.

In der Öffentlichkeit wird Schulabsentismus häufig mit Abwesenheit von der Schule bzw. „Schwänzen“ gleichgesetzt und die Gründe im Desinteresse der SchülerInnen gesucht. Diese intuitive Definition erscheint auf den ersten Blick logisch, jedoch gebietet sich bei genauerer Betrachtung eine differenziertere Herangehensweise. Bedeutsam ist etwa die Frage, ob unter Schulabsentismus nur unentschuldigte oder auch entschuldigte Fehltage gefasst werden und $\mathrm{ab}$ welchem Cut-Off-Wert diese als problematisch angesehen werden müssen oder ob es diesen Wert überhaupt gibt?
Bis heute sind innerhalb der und zwischen den Forschungsdisziplinen unterschiedliche Herangehensweisen, Definitionen und Operationalisierungen des Forschungsgegenstandes „Schulabsentismus" sichtbar.

Ricking und Neukäter (1997) sowie Ricking (2003a) subsumieren beispielsweise unter dem Oberbegriff Schulabsentismus das Schulschwänzen, die Schulverweigerung und das Zurückgehaltenwerden. Das Schulschwänzen im Sinne von Ricking (2003b) geht eher von den SchülerInnen selbst aus, die Eltern wissen häufig nichts von diesen Schulversäumnissen und diese Form der Fehlzeiten besitzen eher Unlust-Charakter (Ricking (2003b): „, angenehmeren Tätigkeiten im außerhäuslichen Bereich nachgehen“). Unter Schulverweigerung versteht er hingegen eine internalisierte emotionale Störungsform, da die SchülerInnen Schwierigkeiten haben, den Unterricht zu besuchen oder sich räumlich der Schule zu nähern. Das Zurückhalten bezieht sich nach Ricking (2003b) im Wesentlichen auf die Erziehungsberechtigten, die das Fernbleiben von der Schule dulden oder sogar unterstützen.

Abweichende Definitionen und weiterführende Untergliederungen finden sich etwa bei Thimm (2000, 2005), Schulze und Wittrock (2001) sowie in der Übersichtsdarstellung von SchreiberKittl und Schröpfer (2002). Dort werden Begriffe wie aktive und passive Schulverweigerung, Schulausstieg, Schulunlust, Schulverdrossenheit, offene Unterrichtsverweigerung, vermeidende Schulverweigerung, Schulangst und Schulmüdigkeit ins Feld geführt.
Trotz der unterschiedlichen Begriffsverwendungen lassen sich wesentliche Gemeinsamkeiten erkennen: Die meisten AutorInnen gehen davon aus, dass hiermit SchülerInnen beschrieben werden, die sich in verschiedenem Ausmaß und auf unterschiedliche Weise dem Unterricht oder der Schule entziehen (Schreiber-Kittl und Schröpfer, 2002). Zu betonen ist, dass es sich im Kern um das gleiche Phänomen handelt und die unterschiedlichen Definitionsverwendungen nicht zu einer einheitlichen Diskussion in der Forschung führen können! Denn wo liegt das tatsächliche und gemeinsame Problem der Schulabsentismus-Debatte? Es liegt in den Konsequenzen, die sich in schulischen Misserfolgen und einem anschließenden sozialen Ausschluss manifestieren können (Ehmann und Rademacker, 2003).

Es fällt auf, dass auf Basis dieser unterschiedlichen Begriffsverwendungen die in Deutschland erhobenen Daten sehr schwer oder gar nicht zu vergleichen sind. Trotzdem soll an dieser Stelle auf einige vorliegende Primärdaten zum Umfang des Schulabsentismus eingegangen werden.

Wetzels et al. (2000a) stellen für Rostock in einer SchülerInnenbefragung fest, dass 10,1\% der SchülerInnen den „,intensiven Schwänzern" zuzuordnen sind, da sie fünf oder mehr unerlaubte Fehltage im Schulhalbjahr aufweisen. Die Analysen aus Berlin (Senatsverwaltung für Bildung, Jugend und Sport, 2005) operationalisieren und betrachten auf Grund von Schulangaben Schulversäumnisse in Kategorien von 1-10, 11-20, 2140 und über 40 Fehltage. Die Ergebnisse zeigen, dass an den Hauptschulen 35,32 \% der Schü- 
lerInnnen mehr als 10 Fehltage (entschuldigte und unentschuldigte) aufweisen. Die Studie aus Schleswig-Holstein (Rat für Kriminalitätsverhütung Schleswig-Holstein, 2007) für das Schulhalbjahr 2004/2005 ergibt auf Basis einer KlassenlehrerInnenbefragung, dass 13,2\% der HauptschülerInnen mehr als 10 (entschuldigte und unentschuldigte) Fehltage aufweisen. Des Weiteren wird für die 9. und 10. Klassen der Hauptschulen berichtet, dass $6,3 \%$ bzw. $5,3 \%$ der SchülerInnen mindestens einen unentschuldigten Fehltag aufzeigen. Wilmers, Enzman, Schäfer, Herbers, Greve und Wetzels (2002, S. 294) berichten hingegen für den Landkreis Friesland (9. und 10. Klasse Hauptschule, Vollerhebung, Selbstauskünfte), dass 56,5 \% der befragten SchülerInnen angeben, mindestens einen Tag unentschuldigt im letzten Halbjahr gefehlt zu haben. Deutlich wird hier eine enorme Diskrepanz zwischen den Angaben für das gesamte Bundesland Schleswig-Holstein und dem Landkreis Friesland. Mögliche Gründe hierfür können in der unterschiedlichen Herangehensweise der Studien gesehen werden. Festzustellen ist allgemein ein Unterschied zwischen Selbstauskünften von SchülerInnen auf der einen und Fremdeinschätzungen von LehrerInnen auf der anderen Seite: So nehmen beispielsweise LehrerInnen in Friesland für $4,4 \%$ der SchülerInnen an, dass diese mindestens fünf Tage unentschuldigt pro Halbjahr fehlen, wohingegen dies $16,4 \%$ der SchülerInnen für sich selbst angeben. Dieser Unterschied wurde auch für andere Großstädte wie z.B. Hamburg und München festgestellt (Wilmers et al., 2002, S. 296). Außerdem fällt bei der Betrachtung der Daten der Schleswig-Holstein-Studie unterteilt nach Klassenstufen auf, dass hier insgesamt $40 \%$ der von der Analyse ausgeschlossen wurden, weil eine Zuordnung der SchülerInnen zu einer bestimmten Klassenstufe nicht möglich war. In diesem Punkt sprechen die AutorInnen dort deshalb auch nur von „indirekten Anhaltspunkten“, die die Ergebnisse liefern.
Ein direkter Vergleich vorhandener Studien ist deshalb allein wegen der Form der Datenerhebungen nicht oder nur schwerlich möglich. Betrachtet man die unerlaubten Fehlzeiten, können diese durch vorgetäuschte Gründe verschleiert werden, indem Versäumnislisten als entschuldigte Fehltage erscheinen und ein verändertes Schulbesuchsverhalten verdecken. Deshalb ist anzunehmen, dass Erhebungen, die sich ausschließlich auf Fremdauskünfte beziehen, das Phänomen des Schulabsentismus eher unterschätzen. Bereits 1997 schlagen daher Dietrich und Freytag vor, die Motivationsstrukturen von SchülerInnen aufzudecken, indem sie Selbsteinschätzungen zu ihren Fehltagen vornehmen. So kann, unabhängig von den Einschätzungen der Lehrkräfte, am besten erfasst werden, wie hoch die unerlaubten Fehlzeiten von SchülerInnen sind und in welchem $\mathrm{Zu}$ sammenhang diese $\mathrm{zu}$ individuellen Problemlagen stehen. Damit stellt sich gleichzeitig die Frage, welche Ursachen und Einflussfaktoren bislang in der Literatur diskutiert werden und welche Ansatzmöglichkeiten für die Entwicklung von präventiven Maßnahmen zu erkennen sind.

\section{Ursachen und Einfluss- faktoren}

In der Literatur werden fünf verschiedene Bereiche genannt, in denen Gründe und aufrecht erhaltene Bedingungen für ein verändertes Schulbesuchsverhalten gesucht werden. Dabei konstatiert Ricking (2003b), dass dieses Phänomen nicht als homogenes Verhaltensmuster verstanden werden kann, sondern Ausdruck und Folge der unterschiedlichen Einflussfaktoren zwischen dem sozialen Umfeld und dem inneren System des/r Schülers/in ist.

\section{Im Bereich des Elternhauses} werden vor allem familiäre Stressoren, wie Beziehungsprobleme der Eltern, häusliche Gewalt, Arbeitslosigkeit, Überbehütung, Einengung, sozioö- konomischer Status, Delinquenzbelastung oder Migrationshintergrund sowie mangelnde Erziehungskompetenz und Erziehungsprobleme als Risikofaktoren für Schulabsentismus herausgestellt ( $\mathrm{vgl}$. Lorenz, 2007; Ricking, 2003; Schreiber-Kittl und Schröpfer, 2002; Stamm 2006).

2. Bei den individuellen Bedingungsfaktoren werden Alter, Geschlecht, Krisen im emotionalen Bereich, (Versagens-) Ängste, Leistungsstörungen, negative Selbstkonzepte, ein geringes Selbstwertempfinden, Affektregulationsstörungen, Verhaltensstörungen und mangelnde Frustrationstoleranz diskutiert (vgl. Lorenz, 2007; Mutzeck, 2005; Reid, 1982, 1999, 2005; Schorr, 1979; Schreiber, 2005; Stamm, 2006; Wilmer und Greve, 2002).

3. Peers können einen Einfluss auf schulabsentes Verhalten haben, wenn selbiges in der PeerGroup beispielsweise als statusfördernd gilt. Es werden aber auch Ablösungsprobleme, Cliquenzusammenhalt, gemeinsames Treffen außerhalb der Schule während den regulären Schulzeiten, Angst vor MitschülerInnen sowie eine geringe Akzeptanz durch Lehrkräfte und MitschülerInnen in der Literatur erwähnt ( $v g l$. Hurrelmann, 2004; Ricking und Neukäter, 1997; Stamm, 2006).

4. Im Bereich des sozialen Umfeldes werden Werteverlust, Orientierungs- und Perspektivlosigkeit sowie Benachteiligung sozial schwacher, problembelasteter Familien als mögliche Risikofaktoren aufgeführt ( $\mathrm{vgl}$. Lorenz, 2007; Mutzeck, 2005).

5. Im Bedingungskontext Schule werden zudem „lebensferne“ schulische Inhalte, ein schlechtes Klassenklima, Probleme mit Lehrkräften, Leistungsprobleme oder Schulängste als mögliche auslösende oder aufrecht erhaltene Bedingungen für einen unregelmäßigen Schulbesuch genannt (vgl. Dietrich und Freytag, 1997; Edelstein, 2002; Hofman-Lun und Michel 2004; Kornmann, 1980; Michel, 2005; Schreiber-Kittl und Schröpfer, 2002; Thimm, 2000).

\section{Delinquenz}

Schulabsentismus kann in einem engen Zusammenhang zur Jugenddelinquenz stehen. So wurde bereits in den 30-er Jahren in den USA die Abwesenheit von der Schule als eine erste Manifestation für eine kriminelle Karriere angesehen (Healy und Bronner, 1933). Im Zweiten Periodischen Sicherheitsbericht der Bundesregierung (BMI/BMJ, 2006) wird zunächst zutreffender Weise darauf verwiesen, dass das gelegentliche unerlaubte Fehlen ein weit verbreitetes und normales Phänomen ist. Gleichzeitig wird der Zusammenhang zwischen häufigen Fehlzeiten und einer deutlich höheren Delinquenzbelastung herausgestellt. Je öfter SchülerInnen demnach die Schule schwänzen, desto stärker sind sie auch in Straftaten involviert. Diese Erkenntnis steht auch im Einklang mit verschiedenen deutsprachigen Studien (z. B. Boers und Reinecke, 2003; Wilmers et al., 2002; Wetzels et al., 2000a). Eine Veröffentlichung aus der Schweiz (Moret, 2006) unterstreicht diese Ergebnisse und stellt fest, dass das Risiko für schwere Diebstähle, Drogendelikte und Gewalt bei SchülerInnen mit häufigerem unerlaubten Fernbleiben von der Schule um das 3,1 bis 4,3 fache steigt. Darüber hinaus wird konstatiert, dass auch das geringe Interesse an der Schule das Risiko für delinquente Verhaltensweisen um das 1,5 bis 2,4 fache erhöht.

\section{Maßnahmen}

Bei den diskutierten Ursachen stellt sich die Frage, welche konkreten Maßnahmen gegen Schulabsentismus tatsächlich ergriffen werden. Dieses wird am Beispiel der schleswig-holsteinischen Studie aufgezeigt (Rat für Kriminalitätsverhütung Schleswig-Holstein, 
2007). Demnach werden sehr häufig, nämlich in mindestens zwei Drittel der untersuchten Fälle, Gespräche mit den SchülerInnen, Gespräche mit den Sorgeberechtigten und Ermahnungen angewandt. Seltener finden Gespräche mit mehreren Beteiligten, eine Zusammenarbeit mit der Jugendhilfe oder Hausbesuche statt. Die von den LehrerInnen genannten Maßnahmen werden häufiger bei Jungen durchgeführt, insbesondere bei Jungen mit Migrationshintergrund. Dabei wird festgestellt, dass die Nachhaltigkeit der Maßnahmen insgesamt als begrenzt eingeschätzt wird.

Da hier überwiegend intervenierend reagiert wird und damit das Kind buchstäblich schon in den Brunnen gefallen ist, können diese Maßnahmen dem Bereich der tertiären Prävention zugerechnet werden. Auf selber Ebene sind Konzepte bei SchulaussteigerInnen anzusiedeln.

Es scheint jedoch möglich und sinnvoll, auch schon früher zu reagieren, um Schulabsentismus von Anfang an zu verhindern oder zu minimieren. Die oben genannten fünf Ursachenbereiche und die konkret diskutierten Einflussgrößen liefern Hinweise für primäre und sekundäre Präventionsmöglichkeiten. So weisen beispielsweise Wilmers und Greve (2002) sowie Neukäter und Ricking (2000) auf einen möglichen $\mathrm{Zu}$ sammenhang zwischen der Qualität des Unterrichts und den Beziehungen zwischen Lehrkörpern und MitschülerInnen als Einflussfaktoren auf die Motivation zum Schulbesuch hin. Interessant erscheinen hier die Ansätze im innerschulischen Bereich, die die Veränderung von Motivation, Selbstwirksamkeit, Klassenklima, Beziehung zu Lehrkräften und lebensfernen schulischen Inhalten in den Mittelpunkt rücken.

Die Ergebnisse einer Regressionsanalyse von Mau (2007), die eine Studie mit 269 HauptschülerInnen der 7.-9. Klassen bzgl. Schulabsentismus in SchleswigHolstein durchführte, zeigen, dass höhere schulische Selbstwirksamkeitserwartungen einen positiven Einfluss auf einen regelmäßigen Schulbesuch haben. Bezieht man die Empfehlungen der SchülerInnen zur Verhinderung unerlaubter Fehlzeiten aus ihrer Studie mit ein, zeigt sich hier ein primärpräventiver Ansatz, um Schulabsentismus zu begegnen. Weit über die Hälfte der SchülerInnen nennen nettere Lehrkräfte, besseren Unterricht und mehr Spaß im Unterricht als Möglichkeiten zur Verringerung schulabsenter Verhaltensweisen. Aus den Ergebnissen der schleswig-holsteinischen Erhebung (Rat für Kriminalitätsverhütung Schleswig-Holstein, 2007) wurden unterschiedliche Vorgehensweisen abgeleitet, so soll z.B. jede Schule ein Handlungskonzept gegen Schulabsentismus entwickeln, das sich auch im Schulprogramm der jeweiligen Schule niederschlagen könnte. Es wird gefordert, dass bei Fernbleiben möglichst sofort gehandelt werden soll, sodass eine erfolgreiche und möglichst schnelle Unterstützung des/der Schüler/in möglich sei. Eine engere Abstimmung zwischen Jugendhilfe auf der einen und der Schule auf der anderen Seite ist erstrebenswert.

Die Entwicklung von präventiven Maßnahmen in den Bereichen der individuellen Bedingungsfaktoren sowie Schule scheint demnach ein vielversprechender Ansatz zu sein, um dem Schulabsentismus erfolgreich begegnen zu können.

\section{Fazit}

Unser Artikel zeigte, dass leider eine Begriffsdiffusion herrscht, die eine Vergleichbarkeit der wissenschaftlichen Datenlage stark erschwert. So scheinen sich teilweise die Ergebnisse unterschiedlicher Studien hinsichtlich Häufigkeit und etwa Geschlechtsunterschiede zu widersprechen, dies scheint aber vor allem am zu Grunde gelegten Kriterium zu liegen.
Für künftige Untersuchungen wäre es insgesamt wünschenswert, zumindest für den Bereich der Schule entschuldigte sowie unentschuldigte Fehltage genau $\mathrm{zu}$ dokumentieren, um längsschnittliche Primärdaten zum Umfang von Schulabsentismus gewinnen zu können.

Um nicht nur tertiäre Prävention bzw. Intervention zu betreiben und so dem „Problem“ Herr zu werden, sondern auch effektive Maßnahmen im Bereich der primären und sekundären Prävention zu entwickeln, ist ein genaues Wissen um die Ursachenzusammenhänge auch im deutschsprachigen Raum notwendig. Dieses Wissen sollte jedoch durch Befragungen der absenten SchülerInnen (und nicht nur der SchulaussteigerInnen!) gewonnen werden, um über die tatsächlichen Beweggründe des Verhaltens Auskunft zu erhalten. So könnte gemeinsam mit SchülerInnen, Eltern und Lehrkörpern an den Erkenntnissen mit den Ziel der Entwicklung von präventiven Maßnahmen angeknüpft werden. Da wie ausgeführt eine enge Verknüpfung zwischen Delinquenz und Schulabsentismus besteht, ist es unerlässlich, die Forschung in diesem Gebiet voranzutreiben, um wirksame Ansätze zur Primärprävention ausmachen zu können.

Inga Mau (Institut für Psychologie, Christian-Albrechts-Universität $\mathrm{zu}$ Kiel)

Sebastian Messer (wissenschaftlicher Mitarbeiter am Institut für Sanktionenrecht und Kriminologie, Christian-Albrechts-Universität zu Kiel)

Katja vom Schemm (wissenschaftliche Mitarbeiterin am Institut für Psychologie, Christian-AlbrechtsUniversität zu Kiel)

\section{Literatur}

BMI / BMJ (2006), Schulschwänzen und Jugenddelinquenz. In BMI / BMJ (Hrsg.), Zweiter Periodischer Sicherheitsbericht (S.370-375). Berlin: Bundesministerium des Innern, Bundesministerium der Justiz.

Boers, K. und Reinecke, J. (2003), Informationen zur zweiten Schülerbefra- gung in Duisburg 2003. Verfügbar unter: URL http://www.uni-bielefeld.de/soz/personen/reinecke/infodu2003.pdf [02.09.2007].

Dietrich, P. und Freytag, R. (1997), „Für das Leben lernen?" - Schulzufriedenheit in Brandenburg. In D. Sturzbecher (Hrsg.), Jugend und Gewalt in Ostdeutschland: Lebenserfahrung in Schule, Freizeit und Familie (S. 113-260). Göttingen: Hogrefe.

Edelstein, W. (1998), Selbstwirksamkeit in der Schulreform, Unterrichtswissenschaft, 26 (2), 100-106.

Ehmann, C. und Rademacker, H. (2003), Schulversäumnisse und sozialer Ausschluss. Vom leichtfertigen Umgang mit der Schulpflicht in Deutschland, Bielefeld: Bertelsmann.

Healy, W. und Bronner, A. F. (1933), How does the school produce or prevent delinquency? Journal of Educational Sociology: A Magazine of Theory and Practice, 6 (8), 450470.

Hildeschmidt, A., Meister, H., Sander, A. und Schorr, E. (1979), Unregelmäßiger Schulbesuch - Verbreitung, Bedingungen, Interventionsmöglichkeiten. Weinheim: Beltz.

Hofmann-Lun, I. und Michel, A. (2004) Schulmüdigkeit und Schulverweigerung - Die Hauptschule unter Hauptverdacht. Diskurs, 14 (1), 28 35.

Hurrelmann, K. (2004), Lebensphase Jugend. Weinheim: Beltz.

Kornmann, R. (1980), Schulschwänzen

- Persönlichkeitsmerkmal oder Symptom verbesserungsbedürftiger Unterrichtsqualität? Psychologie in Erziehung und Unterricht, 27, 240242.

Lorenz, A. (2007), Schulverweigerung. Familie, Partnerschaft, Recht, 13 (12), 33-36.

Mau, I. (2007), Bedingungen und Korrelate des Schulabsentismus. (Unveröffentlichte Diplomarbeit). Christian-Albrechts-Universität zu Kiel: Institut für Psychologie.

Michel, A. (2005), Den Schulausstieg verhindern - Gute Beispiele einer frühen Prävention. München: DJI.

Moret, G. (2006), Der Einfluss der Schullaufbahn auf die Jugendkriminalität. Crimiscope, 31, 1-7.

Mutzek, W. (2005), Schulvermeidung: Begriffe, Sichtweisen, Einflussfaktoren. Schulmagazin, 9, 5-9.

Preuß, E. (1978), Schulschwänzen und Schulverweigerung. In K. J. Klauer (Hrsg.), Sonderpädagogik in allgemeinen Schulen (S. 164-173). Berlin: Marhold.

Rat für Kriminalitätsverhütung Schleswig-Holstein (2007, April), Konzept gegen Schulabsentismus - Jeder Schüler, jede Schülerin zählt. Kiel: Innenministerium des Landes Schleswig-Holstein.

Reid, K. (1982), The self-concept and persistent school absenteeism. British Journal of Eduactional Psychology (52), 179-187. 
Reid, K. (1999), Truancy and Schools. London: Routledge.

Reid, K. (2005), The causes, views and traits of school absenteeism and truancy - An analytic review. Research on Education, 74, 59-82.

Ricking, H. (2003a), Schulabsentismus als Forschungsgegenstand. Oldenburg: bis-Verlag.

Ricking, H. (2003b), Pädagogische Alternativen bei Schulabsentismus. Vortrag auf

der Tagung zum Thema Schulverweigerung, Carl von Ossietzky-Universität

Oldenburg.

Ricking, H. und Neukäter, H. (1997), Schulabsentismus als Forschungsgegenstand. Heilpädagogische Forschung XXIII, 2, 50-70.

Schorr, E. (1979), Ausgewählte Persönlichkeitsfaktoren und Schulbesuchsverhalten. In A. Hildeschmidt, $\mathrm{H}$. Meister, A. Sander \& E. Schorr
(Hrsg.), Unregelmäßiger Schulbesuch - Verbreitung, Bedingungen, Interventionsmöglichkeiten (S.11154). Weinheim: Beltz.

Schreiber-Kittl, M. und Schröpfer, $H$. (2002), Abgeschrieben? Ergebnisse einer empirischen Untersuchung über Schulverweigerer. München: Verlag Deutsches Jugendinstitut.

Schreiber, E. (2005), Nicht beschulbar? Gute Beispiele für den Wiedereinsteig in das systematische Lernen. München: DJI.

Schulze, G. und Wittrock, M. (2001), Abschlussbericht zum Landesforschungsprojekt Schulaversives Verhalten (Band 1). Rostock: Universität Rostock.

Stamm, M. (2006), Schulabsentismus - Anmerkungen zu Theorie und Empirie einer vermeindlichen Randerscheinung schulischer Bildung. Zeitschrift für Pädagogik, 52, 285302.
Senatsverwaltung für Bildung, Jugend und Sport (2005), Weniger Schulversäumnisse in Berlin. Verfügbar unter: URL http://Bildungsklick. de/datei-archiv/19565/Ergebnisse nach Schulart und Jahrgangsstufe.pdf [02.08.2007].

Thimm, K. (2000). Schulverweigerung - Zur Begründung eines neuen Verhältnisses von Sozialpädagogik und Schule. Münster: Votum-Verlag.

Thimm, K. (2005), Schulverweigerung. Ist unsere Schule noch kinder- und jugendgerecht? Verfügbar unter: URL http://www.ganztagsschulverband.de/Download/Schulverw e i g e r u n g T h i m m. d o c [02.08.2007]

Wetzels, P., Mecklenburg, E., Wilmers, N., Enzmann, D. und Pfeiffer, C. (2000a), Gewalterfahrungen, Schulschwänzen und delinquentes Verhalten Jugendlicher in Rostock. Abschlußbericht über die Ergebnisse einer repräsentativen Befragung von Schülerinnen und Schülern. Hannover: KFN.

Wetzels, P., Wilmers, N., Mecklenburg, E., Enzmann, D. und Pfeiffer, C. (2000b), Gewalterfahrungen und Delinquenz Jugendlicher in Delmenhorst. Eine Totalerhebung bei Schülerinnen und Schülern der 9. und 10. Jahrgangsstufe und des Berufsvorbereitungsjahres. Hannover: KFN.

Wilmers, N., Enzmann, D., Schäfer, D., Herbers, K., Greve, W. und Wetzels, P. (2002), Jugendliche in Deutschland zur Jahrtausendwende: Gefährlich oder gefährdet? Ergebnisse wiederholter, repräsentativer Dunkelfelduntersuchungen $\mathrm{zu}$ Gewalt und Kriminalität im Leben junger Menschen 1998 - 2000. Baden-Baden: Nomos.
Die vorhandenen Studien im deutschsprachigen Raum über SchülerInnen, welche unregelmäßig oder selten die Schule besuchen, sind leider kaum vergleichbar. Der Grund ist wohl der, dass schon die jeweiligen Bezugsdaten auf eine wenig einheitliche Weise ermittelt wurden. Die weitere Gewinnung von umfassenden Primärdaten ist selbstredend bedeutsam, um Aussagen über den genauen Umfang und Verlauf von Schulabsentismus treffen zu können. Unbefriedigend ist ferner die Tatsache, dass im Allgemeinen erst dann reagiert wird, wenn das Kind buchstäblich in den Brunnen gefallen ist. Man sollte daher bei diesem Problem weniger tertiäre Prävention bzw. Intervention betreiben, früher reagieren und effektive Maßnahmen im Bereich der primären und sekundären Prävention entwickeln. Hierzu ist aber ein genaues Wissen um die Ursachenzusammenhänge notwendig. Die Forschung sollte hierbei die Betroffenen einbeziehen, etwa durch Befragungen der absenten SchülerInnen hinsichtlich ihrer Beweggründe.

Da auch eine enge Verknüpfung zwischen Delinquenz und Schulabsentismus bzw. zwischen Schulabsentismus und Delinquenz besteht, ist es unerlässlich, mehr über die Gründe für dieses Verhalten zu wissen, um auch hier wirksame
Ansätze zur Primärprävention ausmachen zu können. Die Entwicklung von effektiven Maßnahmenkatalogen ist nur dann möglich, wenn man genügend Informationen sowohl zum Umfang als auch zu den genauen Ursachen hat. Zum jetzigen Zeitpunkt ist festzuhalten, dass die Grundlage aller präventiven Maßnahmen in der allgemeinen Sensibilisierung für dieses Phänomen liegen sollte.

Im Idealfall könnte es sich hierbei um Maßnahmen handeln, die die intrinsische Motivation der SchülerInnen zum regelmäßigen Schulbesuch erhöhen, wobei es hier interessant erscheint, ob und in welchem Umfang Maßnahmen wie beispielsweise die polizeiliche Zuführung einen langfristigen, positiven Effekt erkennen lassen. Wilmers und Greve (2002) sowie Neukäter und Ricking (2000) weisen auf einen möglichen Zusammenhang zwischen der Qualität des Unterrichts und den Beziehungen zwischen LehrerInnen und MitschülerInnen als Einflussfaktoren auf die Motivation zum Schulbesuch hin. Denkbar erscheint hier zudem ein Zusammenhang zwischen Schul-/Klassenklima und Schulbesuchsverhalten.

Im Bereich der Schule-Schülerlnnen-Interaktion können daher vielleicht ein gutes, respektvolles Verhältnis untereinander und ein interessant gestalteter Unterricht eine wichtige Rolle bei der Verringe- rung von Fehlzeiten einnehmen. Dabei sollte vornehmlich eine intensive Zusammenarbeit aller Beteiligten im Bereich der Schule, der Schülerlnnen und des Elternhauses im Mittelpunkt stehen.

Eine genaue Dokumentierung sowie bewusste Wahrnehmung aller Fehlzeiten und Verspätungen ist für eine Einschätzung unerlässlich, da noch keine abschließenden Erkenntnisse darüber vorliegen, ab wann welche Fehlzeiten als problematisch einzuschätzen sind, und welchen genauen Verlauf diese bei den einzelnen SchülerInnen künftig nehmen werden. Zudem liegen möglicherweise auch bei entschuldigten Fehlzeiten individuelle Problemlagen vor (wie beispielsweise das Zurückgehaltenwerden von der Schule), die Schulabsentismus begründen und/oder zu dem Nicht-Erlangen eines Schulabschlusses führen können. Die direkte Ansprache von SchülerInnen und die gleichzeitige Einbeziehung der Eltern zur Lösung der Probleme können gegebenenfalls eine sinnvolle Maßnahme zur Reduzierung von Fehlzeiten sein. Diese Maßnahmen sollten in ein individuell entwickeltes Gesamtkonzept eingebunden werden, welches beispielsweise wie in England als Qualitätsmerkmal von Schulen angesehen werden kann. 